\title{
Solar activities observed with the New Vacuum Solar Telescope
}

\author{
Shuhong Yang ${ }^{1}$ and Jun Zhang ${ }^{1}$ \\ ${ }^{1}$ Key Laboratory of Solar Activity, National Astronomical Observatories, \\ Chinese Academy of Sciences, Beijing 100012, China \\ email: shuhongyang@nao.cas.cn
}

\begin{abstract}
Based on the New Vacuum Solar Telescope observations, some new results about the solar activities are obtained. (1) In the $\mathrm{H} \alpha$ line, a flux rope tracked by filament activation is detected for the first time. There may exist some mild heating during the filament activation. (2) The direct observations illustrate the mechanism of confined flares, i.e., the flares are triggered by magnetic reconnection between the emerging loops and the pre-existing loops and prevented from being eruptive by the overlying loops. (3) The solid observational evidence of magnetic reconnection between two sets of small-scale loops is reported. The successive slow reconnection changes the conditions around the reconnection area and leads to the rapid reconnection. (4) An ensemble of oscillating bright features rooted in a light bridge is observed and given a new name, light wall. The light wall oscillations may be due to the leakage of p-modes from below the photosphere.
\end{abstract}

Keywords. Sun: activity, Sun: atmosphere, Sun: evolution

\section{Introduction}

Solar activities, such as flares, filament eruptions, and coronal mass ejections (CMEs), play an important role in affecting the space environment around the Earth. Therefore, it is necessary for us to study their properties, initiations, and evolution processes. The New Vacuum Solar Telescope (NVST; Liu et al. 2014) located at the Fuxian Solar Observatory (FSO) in China has a diameter of $1 \mathrm{~m}$ and a pure aperture of $980 \mathrm{~mm}$. As the most important facility of the FSO, NVST aims to observe the Sun at high resolution. Three channels are used now to image the Sun: $\mathrm{TiO}$ and $\mathrm{G}$ band are used to observe the fine structures in the photosphere, and $\mathrm{H} \alpha$ is used to image the dynamic structures in the chromosphere. Based on the high tempo-spatial resolution NVST observations, solar activities are investigated and some new results are obtained. In the present paper, we will introduce some recent studies, including the observations of a flux rope tracked by filament activation, the physical mechanism of confined solar flares, the magnetic reconnection between small-scale loops, and the oscillating light wall above a sunspot light bridge.

\section{Observations and Results}

The NVST H $\alpha 6562.8 \AA$ images have a pixel size of $0.16^{\prime \prime}$, and the TiO $7058 \AA$ images have a resolution of $0.05^{\prime \prime} \mathrm{pixel}^{-1}$. They have the same cadence of $12 \mathrm{~s}$. After calibration, the Level 1 images are further reconstructed to Level $1+$ by speckle masking. Besides the NVST observations, the data from the Atmospheric Imaging Assembly (AIA; Lemen et al. 2012) and Helioseismic and Magnetic Imager (HMI; Scherrer et al. 2012) on board the Solar Dynamics Observatory (SDO; Pesnell et al. 2012) and the slit-jaw images (SJIs) 


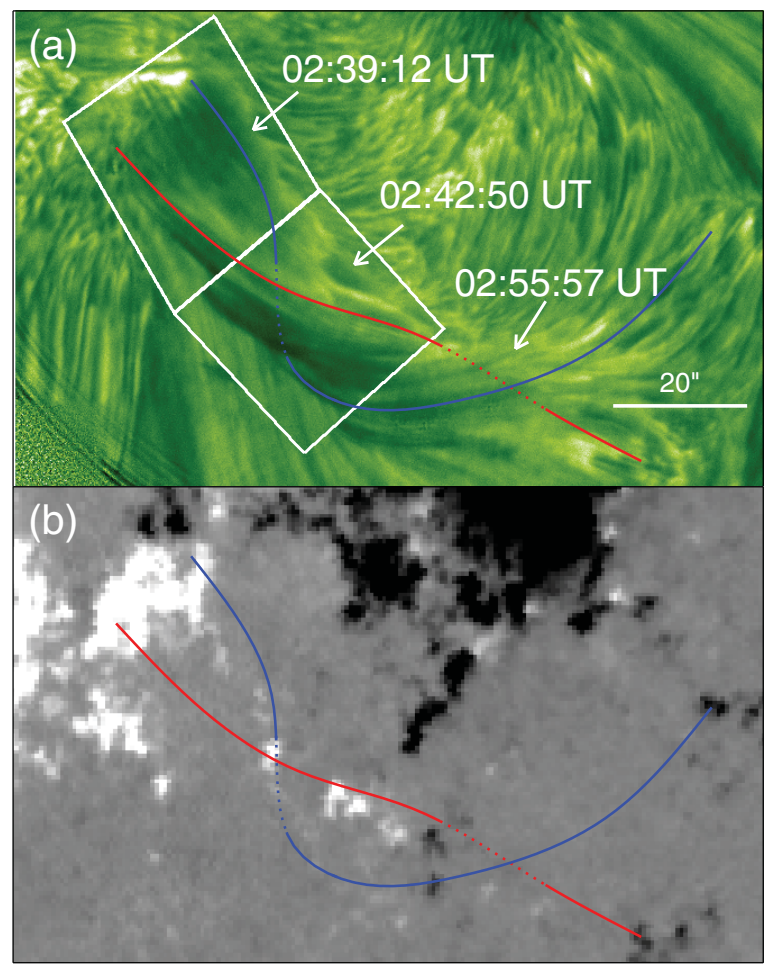

Figure 1. Composite NVST H $\alpha$ image (panel (a)) displaying the flux rope tracked by flowing filament material, and the corresponding HMI photospheric magnetogram (panel (b)) on 2013 February 1. The red and blue curves outline the twist configuration of the flux rope.

from the Interface Region Imaging Spectrograph (IRIS; De Pontieu et al. 2014) are also used. The multi-channel images of the AIA have a pixel size of $0 .{ }^{\prime \prime} 6$ and a cadence of 12 s. The spatial and temporal resolutions of the SJIs are as high as $0.166^{\prime \prime}$ pixel $^{-1}$ and 7 $\mathrm{s}$, respectively.

According to some previous studies, flux ropes are often observed as hot channels in the inner corona (e.g., Zhang et al. 2012; Cheng et al. 2013). Based on the NVST H $\alpha$ data and combined with the simultaneous AIA observations for the first time, we study in detail a flux rope tracked by filament activation on 2013 February 1 (Yang et al. 2014a). The filament material initially fills in a section of the flux rope, and then is activated by magnetic cancellation. The activated filament material flows along helical threads, tracking the twisted structure of the flux rope (Fig. 1(a)). The sub-regions outlined by two quadrangles are the $\mathrm{H} \alpha$ images obtained at 02:39:12 UT and 02:42:50 UT, respectively, and the rest of the background is the image obtained at 02:55:57 UT. The entire flux rope appears as a twisted structure connecting the positive fields (the white patches in Fig. 1(b)) at the northeast and the negative fields (the black patches) at the southwest. The flux rope has an approximate length of $75 \mathrm{Mm}$. Moreover, the detailed study of AIA images shows that, during the filament activation process, there may exist some mild heating of cool filament material to coronal temperatures.

Solar flares as one of the most energetic phenomena in the solar atmosphere can be classified into two types, "eruptive flares" which are associated with CMEs and "confined flares" without CMEs. Using the $\mathrm{H} \alpha$ observations from the NVST, we focus on the fine structures and evolution of three confined flares occurring on 2013 October 12 


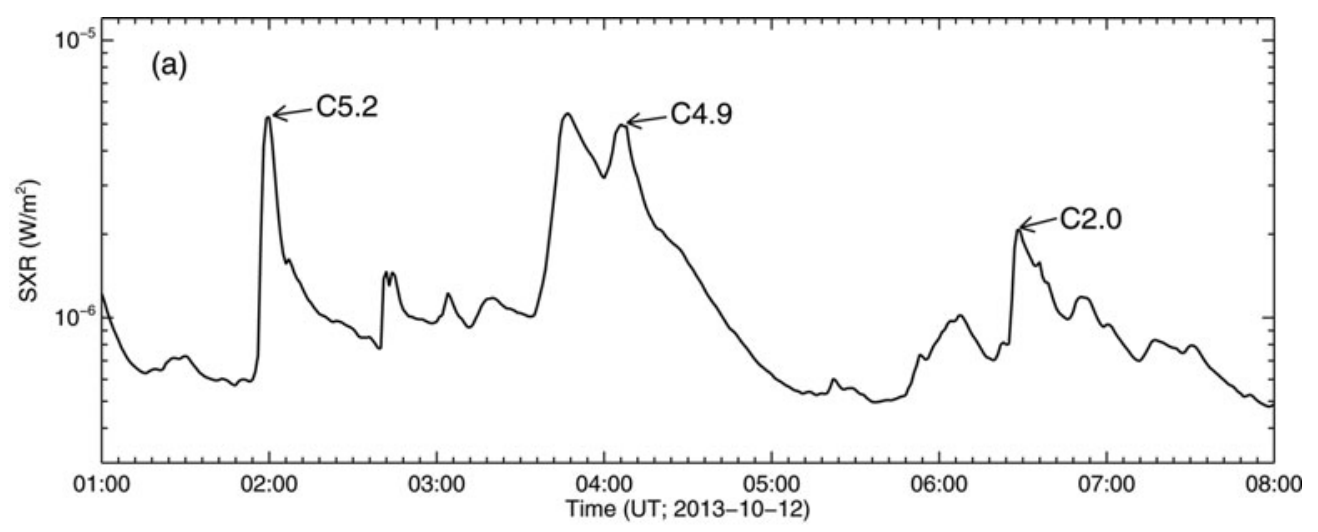

(b) NVST H $01: 59: 03$ UT

(c) AIA $131 \AA$ A 01:59:08 UT

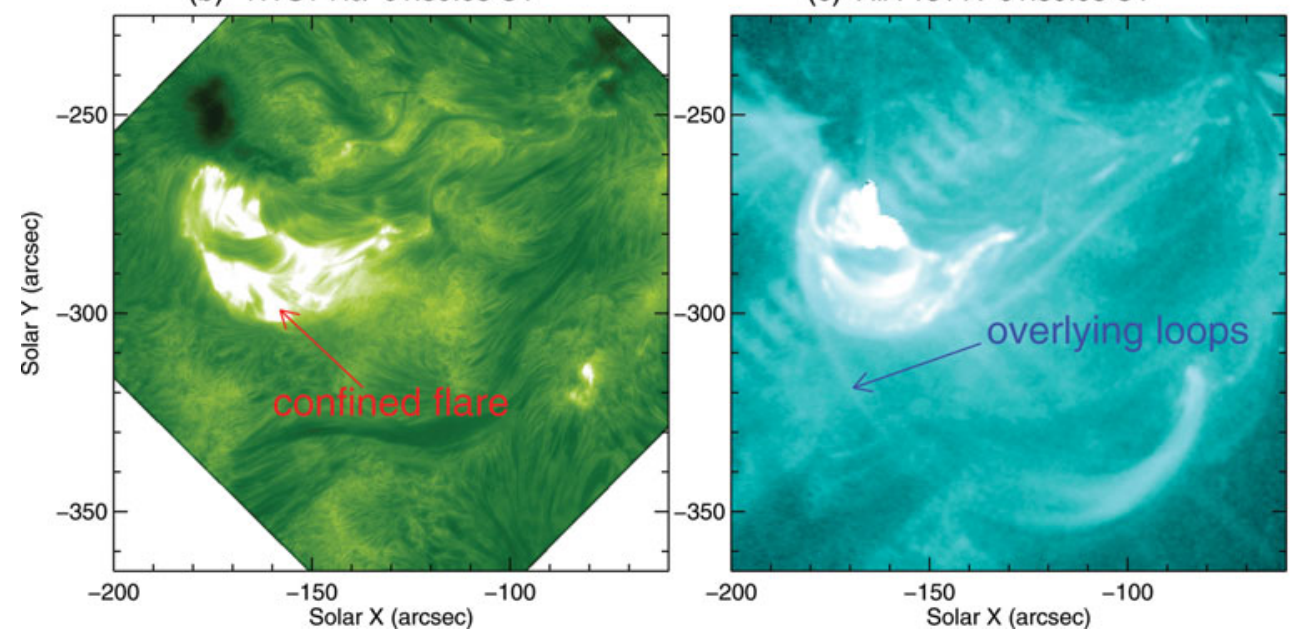

Figure 2. Panel (a): Variation of the GOES soft X-ray flux revealing the evolution of three flares on 2013 October 12. Panels (b) and (c): NVST H $\alpha$ image displaying one of the three confined flares, and AIA $131 \AA$ image showing the corresponding overlying loops, respectively.

(Yang et al. 2014b). The flares take place successively and have comparable classes (C5.2, C4.9, and C2.0), as shown in Fig. 2(a). The three confined flares take place at the same location and have similar morphologies, so can be termed "homologous confined flares". One confined flare is displayed in Fig. 2(b). The bright structure is the C5.2 flare observed in the $\mathrm{H} \alpha$ line. In the $131 \AA$ image (Fig. 2(c)), many large-scale overlying loops can be clearly seen. The average length of the overlying loops is about $130 \mathrm{Mm}$. The direct observations illustrate the mechanism of confined flares, i.e., the flares are triggered by magnetic reconnection between the emerging loops and the pre-existing loops and prevented from being eruptive by the overlying loops.

Magnetic reconnection is a fundamental physical process in conductive plasma. When magnetic reconnection takes place, magnetic energy is released and converted to the kinetic and thermal energy of plasma, which is often considered to be the energy source of solar flares and many types of outbursts (e.g., Yuan et al. 2009; Yang et al. 2011). According to most theories, there exist topological changes, sudden brightenings, and strong outflows during the magnetic reconnection (e.g., Parker 1957). Using the high tempospatial resolution NVST H $\alpha$ observations on 2014 February 3, we give solid evidence of 


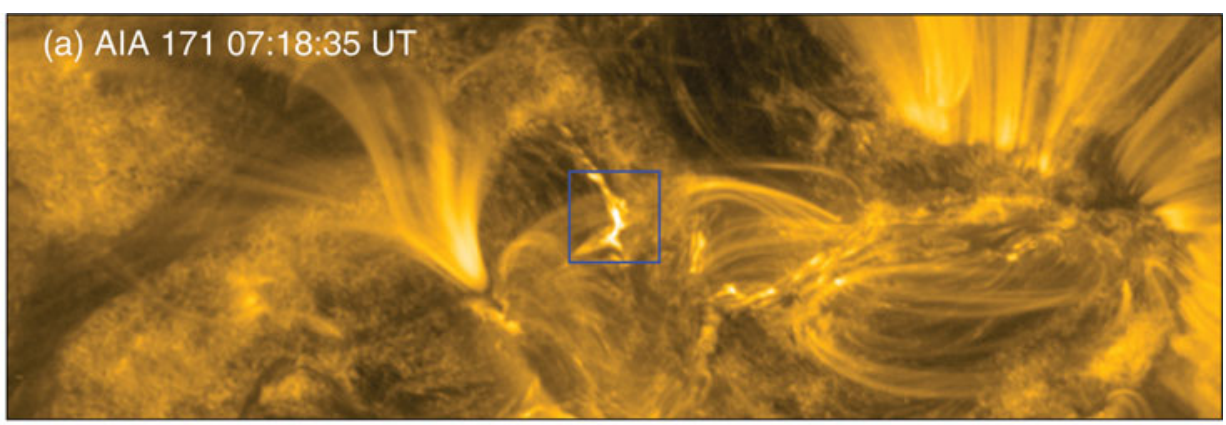

$\begin{array}{lll}\text { (b) NVST H } \alpha \text { 07:17:03 UT } & \text { (c) NVST H } \alpha \text { 07:18:52 UT } & \text { (d) NVST H } \alpha \text { 07:23:31 UT }\end{array}$

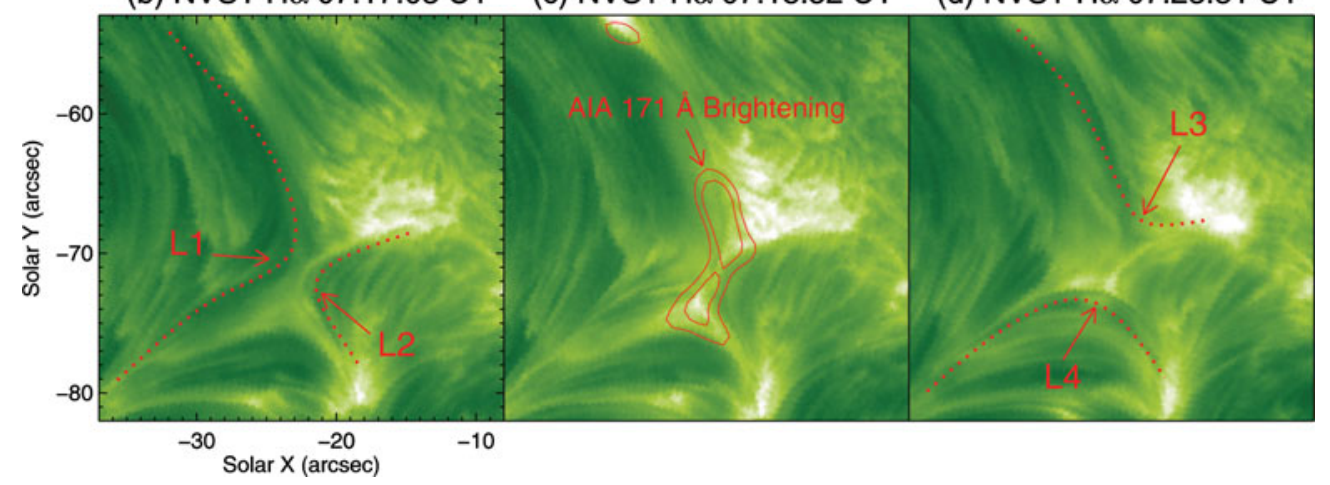

Figure 3. AIA $171 \AA$ image (panel (a)) showing the overview of the area where the magnetic reconnection occurs, and NVST $\mathrm{H} \alpha$ images (panels (b)-(d)) displaying the evolution process of the rapid reconnection. The box in panel (a) outlines the field-of-view of panels (b)-(d). The dotted curves denoted by arrows "L1" and "L2" in panel (b) outline the initial loops before reconnection, and the curves indicated by arrows "L3" and "L4" in panel (d) outline the newly formed loops after reconnection. The red curves in panel (c) are the contours of AIA $171 \AA$ brightness.

magnetic reconnection between two groups of small-scale loops (Yang et al. 2015a). The magnetic reconnection takes place at the edge of NOAA 11967 (see Fig. 3(a)). The loops involved into the reconnection are labeled "L1" and "L2" and outlined by the dotted curves in Fig. 3(b). Loops "L1" and "L2" move toward each other and eventually reconnect (Fig. 3(c)). When magnetic reconnection takes place, an obvious brightening can be observed, especially in $171 \AA$ line (see the contours in panel (c)). Moreover, apparent material ejections outward from the reconnection region are also observed. Due to the reconnection, two sets of new loops ("L3" and "L4" in Fig. 3(d)) are formed, and then move away from each other. Our observations are highly consistent with the predictions by the models of magnetic reconnection. In addition, we find that the reconnection process includes two steps: a slow step and a rapid step. The slow step has a duration of more than several tens of minutes and the rapid step lasts for only about three minutes. We suggest that the successive slow reconnection changes the conditions around the reconnection area and thus leads to the rapid reconnection. In the current event, the brightening region between the approaching loops has a thickness of about $420 \mathrm{~km}$ and a length of $1.4 \mathrm{Mm}$. It seems that that a current sheet might be embedded inside this brightening structure.

NOAA 12192 is a quite huge active region which appeared in the weak solar cycle 24 . NVST TiO $7058 \AA$ images can resolve the fine structures in the photosphere. As displayed 
(a) NVST TIO 05:50:03 UT

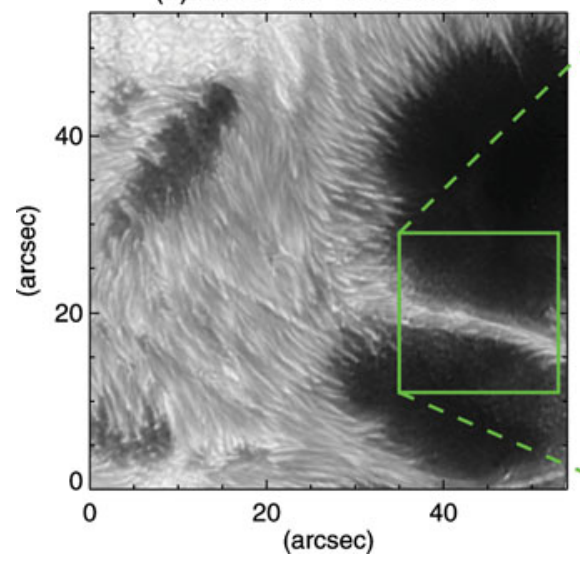

(b) IRIS $1330 \AA$ Å 05:50:17 UT

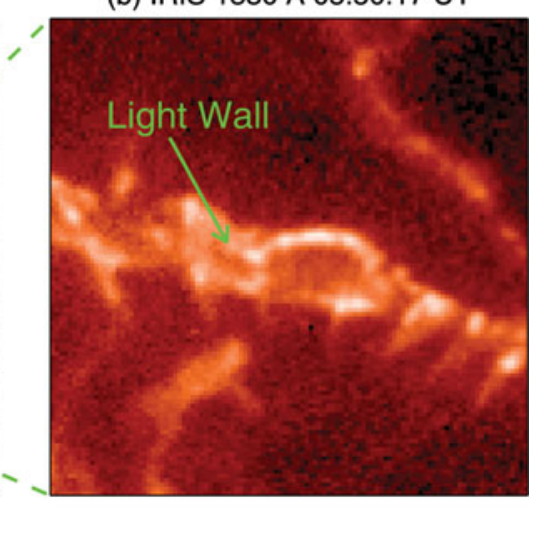

Figure 4. NVST TiO $7058 \AA$ image (panel (a)) showing the light bridge on 2014 October 25, and IRIS SJI $1330 \AA$ image (panel (b)) displaying the light wall rooted in the light bridge.

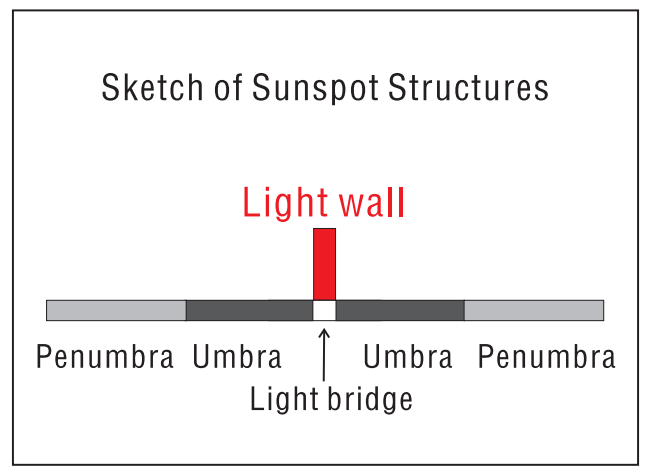

Figure 5. Sketch of sunspot structures based on the new observations.

in Fig. 4(a), there is a distinct strong light bridge crossing the umbra of the sunspot in the TiO image observed on 2014 October 25. Fig. 4(b) is a $1330 \AA$ image from the IRIS, and we can see that many bright structures are rooted in the light bridge. We give the ensemble of these bright features a new name, light wall (denoted by the green arrow; Yang et al. 2015b). The light wall is brighter than the surrounding areas, and the wall top is much brighter than the wall body. The wall top moves upward and downward, i.e., oscillating continuously. The mean deprojected height, amplitude, oscillation velocity, and period are about $3.6 \mathrm{Mm}, 0.9 \mathrm{Mm}, 15.4 \mathrm{~km} \mathrm{~s}^{-1}$, and 3.9 minutes, respectively. The oscillations of the light wall may be caused by the leakage of p-modes from below the photosphere. The brightness enhancement of the light wall top implies that there may exist some kind of atmospheric heating, e.g., via the magneto-acoustic waves or the continuous small-scale reconnection. As the previous traditional knowledge, a mature sunspot is mainly consisted of umbra, penumbra, and light bridge. Our observations show that light wall is also a basic structure of sunspot, as illustrated in Fig. 5.

\section{Summary}

- A flux rope tracked by filament activation is observed in $\mathrm{H} \alpha$ line for the first time, and there exists some mild heating during the filament activation. 
- The direct observations illustrate the physical mechanism of confined flares, i.e., the flares are triggered by magnetic reconnection between the emerging loops and the pre-existing loops and prevented from being eruptive by the overlying loops.

- The high tempo-spatial resolution observations present the solid evidence of smallscale magnetic reconnection, which is highly consistent with the magnetic reconnection models.

- An ensemble of oscillating bright features rooted in a light bridge is observed and named with a new term, light wall.

\section{Acknowledgements}

This work is supported by the National Natural Science Foundations of China (11203037, 11533008, 11221063, 11373004, and 11303049), the Strategic Priority Research Program-The Emergence of Cosmological Structures of the Chinese Academy of Sciences (No. XDB09000000), and the Youth Innovation Promotion Association of CAS (2014043).

\section{References}

Cheng, X., Zhang, J., Saar, S. H., \& Ding, M. D. 2012, ApJ, 761, 62

De Pontieu, B., Title, A. M., Lemen, J. R., et al. 2014, Solar Phys., 289, 2733

Lemen, J. R., Title, A. M., Akin, D. J., et al. 2012, Solar Phys., 275, 17

Liu, Z., Xu, J., \& Gu, B.-Z., et al. 2014, RAA, 14, 705

Parker, E. N. 1957, JGR, 62, 509

Pesnell, W. D., Thompson, B. J., \& Chamberlin, P. C. 2012, Solar Phys., 275, 3

Scherrer, P. H., Schou, J., Bush, R. I., et al. 2012, Solar Phys., 275, 207

Yang, S., Zhang, J., Li, T., \& Liu, Y. 2011, ApJL, 732, L7

Yang, S., Zhang, J., Liu, Z., \& Xiang, Y. 2014a, ApJL, 784, L36

Yang, S., Zhang, J., \& Xiang, Y. 2014b, ApJL, 793, L28

Yang, S., Zhang, J., \& Xiang, Y. 2015a, ApJL, 798, L11

Yang, S., Zhang, J., Jiang, F., \& Xiang, Y. 2015b, ApJL, 804, L27

Yuan, F., Lin, J., Wu, K., \& Ho, L. C. 2009, MNRAS, 395, 2183

Zhang, J., Cheng, X., \& Ding, M.-D. 2012, NatCo, 3, 747 